\title{
Monitoring New Symptoms After COVID-19 Infection Among Primary Care Patients in New York City
}

\author{
Kelly Terlizzi, BA, Eric Kutscher, MD, and Yuliya Yoncheva, PhD
}

Introduction: COVID-19 affects multiple organ systems causing substantial long-term morbidity. The implications of the Post-Acute Sequelae of SARS-CoV-2 infection, particularly for primary care, remain unknown. This cross-sectional study examines new symptoms reported at primary care encounters during three post-acute follow-up intervals after initial SARS-CoV-2 infection.

Methods: Electronic health record data from the NYU Langone COVID Deidentified Dataset were queried for adults with a positive SARS-CoV-2 PCR test, and then restricted to those with a new ICD-10-CM code documented at a post-acute COVID-related primary care follow-up $>14$ days after testing positive. New diagnoses and the corresponding Clinical Classifications Software Refined categories were assessed at the following intervals: 0.5-3 months ("subacute"), 3-6 months ("prolonged"), and 6-9 months ("persistent").

Results: Out of 3,154 patients, a new ICD-10-CM code was documented among 499 patients ( $\sim 16 \%)$. Respiratory complaints, including cough, shortness of breath, dyspnea, and hypoxemia, were most common. Malaise and fatigue were reported consistently among 10-13\% of patients at all three time-intervals. Musculoskeletal pain, circulatory symptoms, and sleep-wake disorders were also observed at primary care follow-up.

Conclusion: This cross-sectional study provides support of a post-acute COVID syndrome, demonstrating that patients continue to experience symptoms after the acute infection period. Extensive follow-up data allowed for examining new symptoms up to 9 months after initial SARS-CoV-2 infection. Understanding of the course of multi-organ post-acute sequelae is restricted by cross-sectional study design limitations. Standardized, sequelae-related ICD-10-CM codes to specify the type and duration of post-acute COVID-related symptoms would enable better monitoring of the growing number of SARSCoV-2 infection survivors. (J Am Board Fam Med 2021;34:1014-1016.)

Keywords: COVID-19, Cross-Sectional Studies, New York, Primary Health Care

\section{Introduction}

Coronavirus disease-2019 (COVID-19) is now recognized as a multi-organ disease. ${ }^{1}$ Long-term complications, collectively termed "post-acute sequelae of

This article was externally peer reviewed.

Submitted 23 March 2021; revised 17 June 2021; accepted 18 June 2021.

From the Department of Population Health, NYU Grossman School of Medicine, New York, NY (KT); Internal Medicine, NYU Langone Health, New York, NY (EK); Department of Child and Adolescent Psychiatry, New York University Grossman School of Medicine, New York, NY (YY).

Funding: None.

Conflict of interest: None.

Corresponding author: Yuliya Yoncheva, $\mathrm{PhD}$, Department of Child and Adolescent Psychiatry, New York University Grossman Shool of Medicine, One Park Avenue, $7^{\text {th }}$ floor, New York, NY 10016, Telephone: 646-754-5147 (E-mail: Yuliya. Yoncheva@nyulangone.org).
COVID-19 infection" or "post-acute COVID-19 syndrome," span a gamut of organ system sequelae ${ }^{2-4}$ with implications for primary care remaining largely unknown. This cross-sectional study describes new symptoms among primary care patients at 3 followup intervals after initial COVID-19 infection.

\section{Methods}

We queried electronic health record data from the New York University (NYU) Langone COVID Deidentified Dataset on January 10, 2021, for adults with $\geq 1$ encounter across NYU Langone Health $>14$ days before and $>14$ days after their earliest positive SARS-CoV-2 polymerase chain reaction (PCR) test. A subset where $\geq 1$ International Classification of Disease, Tenth Version, Clinical Modification (ICD-10$\mathrm{CM}$ ) code was first documented at a COVID-related 
Table 1. Characteristics of Patients Presenting with New Symptoms Following Acute Coronavirus Disease19 (COVID-19; $N$ = 499)

\begin{tabular}{|c|c|}
\hline & Patients \\
\hline & $\mathrm{n}(\%)$ \\
\hline \multicolumn{2}{|l|}{ Sex } \\
\hline Male & $213(42.7)$ \\
\hline Female & $286(57.3)$ \\
\hline \multicolumn{2}{|l|}{ Age, years } \\
\hline Estimated Mean $\pm \mathrm{SD}$ & $56.1 \pm 16.6$ \\
\hline 18 to 32 & $49(9.8)$ \\
\hline 33 to 47 & $109(21.8)$ \\
\hline 48 to 62 & $154(30.9)$ \\
\hline 63 to 77 & $132(26.5)$ \\
\hline$\geq 78$ & $55(11.0)$ \\
\hline \multicolumn{2}{|l|}{ Race } \\
\hline White & $240(48.1)$ \\
\hline Black & $110(22.0)$ \\
\hline Asian & $11(2.2)$ \\
\hline Other* & $112(22.5)$ \\
\hline Unknown & $26(5.2)$ \\
\hline \multicolumn{2}{|l|}{ Ethnicity } \\
\hline Hispanic & $157(31.5)$ \\
\hline Non-Hispanic & $316(63.3)$ \\
\hline Unknown & $26(5.2)$ \\
\hline \multicolumn{2}{|l|}{ Acute COVID-19 } \\
\hline Hospitalized & $243(48.7)$ \\
\hline Intensive Care Unit & $31(6.2)$ \\
\hline Intubation & $25(5.0)$ \\
\hline Dialysis & $14(2.8)$ \\
\hline Treatment (Dexamethasone or Remdesivir) & $25(5.0)$ \\
\hline$\geq 1$ Primary Care Visits & $174(34.9)$ \\
\hline Telemedicine & $151(30.3)$ \\
\hline \multicolumn{2}{|l|}{ Post-Acute COVID-19 Primary Care Visits ${ }^{\dagger}$} \\
\hline 1 & $361(72.4)$ \\
\hline 2 & $76(15.2)$ \\
\hline$\geq 3$ & $62(12.4)$ \\
\hline \multicolumn{2}{|l|}{ Pre-existing Conditions } \\
\hline Hypertension & $262(52.5)$ \\
\hline Diabetes & $134(26.9)$ \\
\hline Obesity & $103(20.6)$ \\
\hline Asthma & $85(17.0)$ \\
\hline COPD & $42(8.4)$ \\
\hline HIV Infection & $6(1.2)$ \\
\hline
\end{tabular}

COPD, chronic obstructive pulmonary disease; ICD-10-CM, International Classification of Disease, Tenth Version, Clinical Modification; HIV, human immunodeficiency virus; PCR, polymerase chain reaction; $\mathrm{SD}$, standard deviation.

*Other race includes American Indian or Alaska Native, Other Pacific Islander, or unspecified Other race by patient report.

${ }^{\dagger}$ Number of COVID-19 related primary care visits for a new symptom (defined by ICD-10-CM codes which do not appear during the patient's lookback and first appear between 15 days and 9 months after the patient's first positive SARS-CoV-2 PCR test). primary care follow-up visit was further analyzed. The mean lookback period was 74 days (median, 60; interquartile range $[\mathrm{IQR}], 38$ to 85 ; $\max , 341)$. Primary care encounters included those at departments of Internal Medicine, Family Medicine, or Primary Care, as well as telemedicine. Encounters accompanied by ICD-10-CM code U07.1 were considered "COVID-related." ICD-10-CM codes were categorized using the Clinical Classifications Software Refined (CCSR) tool. The 15 most frequent CCSR categories were reported at 3 follow-up intervals ${ }^{1}: 0.5$ to 3 months ("subacute"), 3 to 6 months ("prolonged"), and 6 to 9 months ("persistent").

\section{Results}

Of the 3154 patients with pre- and post-COVID encounters, 499 presented with $\geq 1$ new symptom at follow-up with primary care (Table 1). Of those, 419 $(84 \%)$ received care in the subacute interval, 95 $(19 \%)$ in the prolonged interval, and $45(9 \%)$ in the persistent interval, with similar sample characteristics across intervals. On average, each patient's new symptoms were characterized by 2 distinct CCSR categories (median, 2; IQR, 1 to 4; max, 12). Overall, respiratory system complaints were most common ( $27 \%$ of 419 in the subacute, $18 \%$ of 95 in the prolonged, and $11 \%$ of 45 in the persistent interval), of which cough, shortness of breath, dyspnea, and hypoxemia were most frequent (Figure 1). New asthma diagnoses were also found. Malaise and fatigue consistently emerged among $10 \%$ to $13 \%$ of patients, and vitamin D deficiency in $\sim 11 \%$. Musculoskeletal pain was documented among $5 \%$ of patients in the subacute, $13 \%$ in the prolonged, and none in the persistent interval. Nervous system complaints were most often gait- and mobility-related; sleep-wake disorders most often were insomnia. Circulatory symptoms, for example, palpitations and tachycardia, were reported across all time intervals. Hyperlipidemia, type 2 diabetes, and chronic kidney disease were addressed at a higher percentage of encounters within the persistent interval than the subacute interval. Acute pulmonary emboli, thromboembolism, and acute kidney disease were fairly infrequent in follow-up.

\section{Discussion}

In an early COVID-19 epicenter, we found that $\sim 16 \%$ of patients infected with COVID-19 sought 
Figure 1. Top 15 Clinical Classifications Software Refined (CCSR) categories for which patients sought care through their primary care physician. Percentages are reported out of the number of patients with $\geq 1$ newly documented symptom seeking care at each time interval following acute Coronavirus disease-19 (COVID-19).

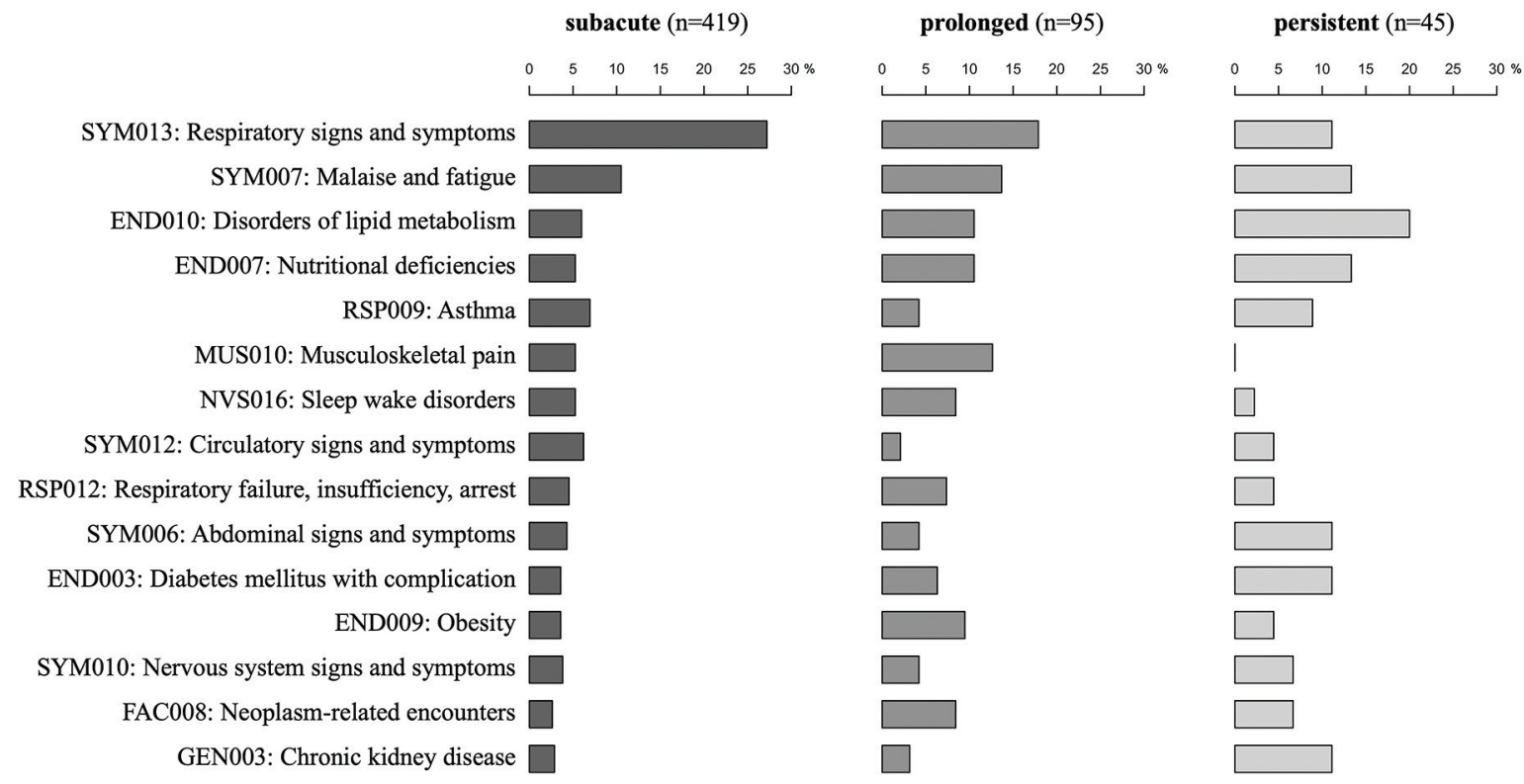

primary care for a new, COVID-related concern within 9 months after initial infection. Overall, the most common new symptoms included respiratory concerns, musculoskeletal pain, malaise, and fatigue. Multi-organ sequelae of COVID-19 were documented, suggesting persistent or delayed longterm complications.

Extensive follow-up data allowed characterization of primary care encounters in biologically meaningful subacute, prolonged, and persistent post-infection intervals. While this exploration yielded preliminary support for investigating postacute sequelae across different timeframes, it also highlighted the limitations of this cross-sectional study. Discerning delayed presentation of postacute COVID symptoms from prolonged or persistent symptoms was impossible without longitudinal data. Moreover, our methods depended on patients' utilization of primary care and departments' coding of U07.1, likely underestimating the presence of COVID-19-related symptoms.

Further monitoring of symptoms would benefit from the creation of standardized, sequelae-related ICD-10-CM codes, for example, "Symptomatology
Secondary to Prior COVID-19 infection" with associated modifiers for subtypes (eg, respiratory, musculoskeletal, fatigue, unspecified) and durations (subacute, prolonged, persistent). The current findings motivate systematic prospective investigations into the lingering effects of COVID-19 and its pernicious impact on morbidity.

To see this article online, please go to: http://jabfm.org/content/ 34/5/1014.full.

\section{References}

1. Nalbandian A, Sehgal K, Gupta A, et al. Post-acute COVID-19 syndrome. Nat Med 2021;27:601-15.

2. Nasserie T, Hittle M, Goodman SN. Assessment of the frequency and variety of persistent symptoms among patients with COVID-19: a systematic review. JAMA Netw Open 2021;4:e2111417.

3. Huang C, Huang L, Wang Y, et al. 6-month consequences of COVID-19 in patients discharged from hospital: a cohort study. The Lancet 2021;397: $220-32$.

4. Carfi A, Bernabei R, Landi F, Gemelli Against COVID-19 Post-Acute Care Study Group. Persistent symptoms in patients after acute COVID-19. JAMA 2020;324:603-5. 